\title{
Der Einbau von Krypton in Quarzkristallen
}

\author{
Ein Beitrag zur Bestimmung des Transformationspunktes Quarz/Cristobalit*
}

Von R. SIzManN und W. RupP

\begin{abstract}
Aus dem Laboratorium für Technische Physik der Technischen Hochschule München
(Z. Naturforschg. 16 a, 861-869 [1961] ; eingegangen am 13. Februar 1961)
\end{abstract}

Herrn Prof. Dr. N. Rrehl zum 60. Geburtstag gewidmet

\begin{abstract}
Beim Erhitzen von Quarzkristallen in einer Gasatmosphäre von radioaktivem Krypton-85 wird hauptsächlich während der Phasenumwandlung $\beta$-Quarz $\rightarrow \beta$-Cristobalit Krypton in den Kristallen aufgenommen. Die damit verbundene nahezu sprunghafte Zunahme der Radioaktivität der Kristalle gestattete eine genaue Bestimmung des Transformationspunktes $\left(930 \pm 15^{\circ} \mathrm{C}\right)$.

Bei den Umwandlungen $\alpha \gtrless \beta$-Cristobalit $\left(270^{\circ} \mathrm{C}\right)$ und $\alpha \gtrless \beta$-Quarz $\left(575^{\circ} \mathrm{C}\right)$ wird dagegen von den Kristallen kein Krypton aufgenommen.

Die Ursache dieses Verhaltens wird auf den unterschiedlichen Typ der Phasenumwandlungen zurückgeführt (Gitterneubau bei Quarz $\rightarrow$ Cristobalit; Gitterumbau bei den $\alpha \gtreqless \beta$-Transformationen).
\end{abstract}

Quarzkristalle, in einer Kryptonatmosphäre erhitzt, nehmen das Gas fast ausschließlich bei der nahe $900{ }^{\circ} \mathrm{C}$ einsetzenden Umwandlung $\beta$-Quarz $\rightarrow$ $\beta$-Cristobalit in sich auf. So läßt sich der bisher schwierig und nur ungenau faßbare Transformationspunkt des trägen $\beta$-Quarz $\rightarrow \beta$-Cristobalit-Überganges bestimmen. Dieser Übergang von hexagonaler zu kubischer Symmetrie ist eine Phasenumwandlung, bei der sich die Gitterbausteine unter Bindungslösung zu einem andersartigen Gittertyp umgruppieren müssen (Phasenumwandlung mit Gitterneubau). Bei den sog. Hoch-Tief-Umwandlungen ( $\alpha \rightleftarrows \beta$-Quarz oder $\alpha \rightleftarrows \beta$-Cristobalit) findet kein Kryptoneinbau statt. Bei diesen Übergängen werden nur Valenzwinkel verdreht (Phasenumwandlung mit Gitterumbau, Übergang von tetragonaler zu kubischer Symmetrie).

Im Vergleich zu den bekannten Diffusions- und Phasentransformationsmessungen mit kurzlebiger, natürlich radioaktiver Emanation ${ }^{1}-H_{\text {AHNsches }}$ Emanierverfahren - ergeben sich bei der Verwendung des langlebigen Krypton-85 methodisch neue Wege.

\section{Experimentelle Anordnung}

Für die Diffusions- und Einbauuntersuchungen mit radioaktivem Krypton in Festkörperproben diente eine Apparatur gemäß Abb. 1, die folgende Arbeitsgänge gestattete: Evakuieren der Probe;

* Auszugsweise vorgetragen auf der Tagung der Physikalischen Gesellschaft in Bayern 25. - 28. April 1960, Erlangen. Siehe „Phys. Verhandl.“11, 49 [1960].
Aufheizen der Probe bis maximal $1600^{\circ} \mathrm{C}$; Einlassen von radioaktivem Krypton für eine bestimmte Versuchszeit; Abführen des Kryptons zur wiederholten Verwendung. Zur Vermeidung von Verlusten an radioaktivem Gas wurde mit STock-Quecksilberventilen gearbeitet.

Um das Krypton in die Anlage einzulassen und es daraus wieder zu entfernen, wurde die starke, temperaturabhängige Adsorptionsfähigkeit von Aktivkohle ausgenutzt.

$10 \mathrm{~g}$ grobgekörnte Aktivkohle wurden in dem Jenaer Glasrohr (2) 10 Stdn. lang unter Vakuum (rd. 10 Torr) bei $400^{\circ} \mathrm{C}$ ausgeheizt. In die vorher evakuierte Apparatur werden dann einige Torr Krypton gefüllt und die Kohle in flüssiger Luft abgekühlt. Dabei wird das Edelgas praktisch vollständig adsorbiert. Aus Sicherheitsgründen wird beim Abkühlen das mit der Kohle gefüllte Glasrohr (2) mit einem Kupferbecher umgeben.

Das Krypton wird bei Bedarf aus der Aktivkohle durch Erhitzen ausgetrieben, wofür $\mathrm{rd} .300{ }^{\circ} \mathrm{C}$ genügen.

In Vorversuchen wurde diese „Pumpwirkung“ der Aktivkohle eingehend untersucht. Abb. 2 gibt einige Adsorptionskurven wieder. Man erkennt, daß bei Zimmertemperatur noch ein großer Teil des Gases an der Kohle adsorbiert bleibt und erst über $300{ }^{\circ} \mathrm{C}$ die Desorption vollständig wird. Nach dem Abkühlen der Kohle in flüssiger Luft war auch mit der empfindlichen Radioaktivitätsmessung in der Anlage bei (6) kein Krypton mehr feststellbar.

Die Quarzprobe befindet sich in einem Rohr (rd. $40 \mathrm{~cm}$ lang, $7 \mathrm{~mm} \varnothing$ ) aus hochschmelzender Pythago-

\footnotetext{
1 K. E. Zimen, Z. physik. Chem. B 37, 231 [1937].
} 


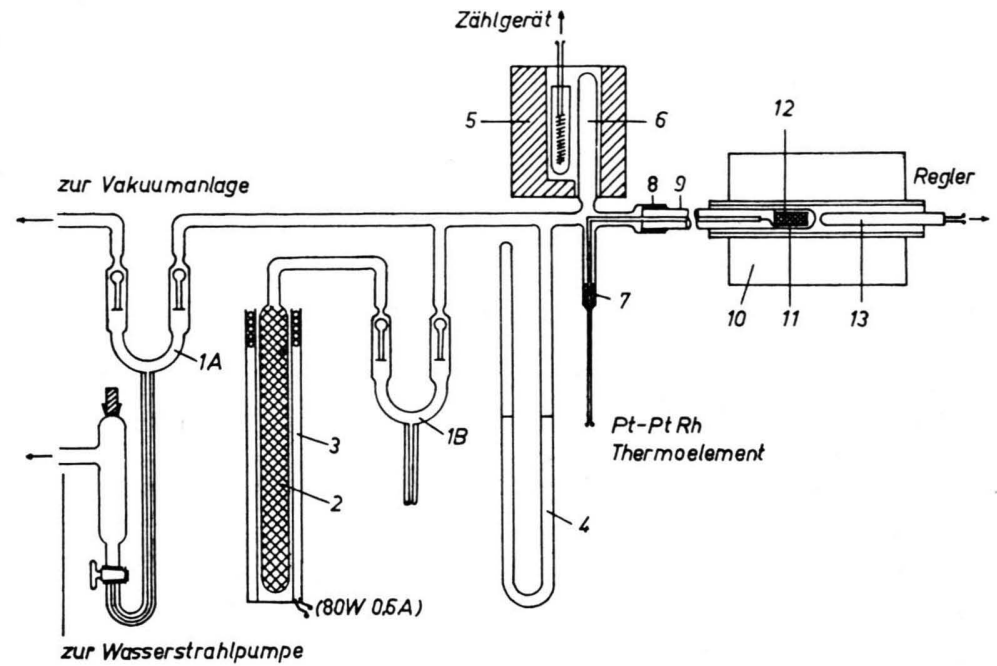

\begin{abstract}
Abb. 1. Apparatur zu Diffusionsuntersuchungen mit Krypton-85. $1 \mathrm{~A}$ und 1 B: Sтоск-Ventile; 2 : Kryptonbehälter mit Aktivkohlefüllung (Jenaer Glas) ; 3: Rohrofen zum Ausheizen der Aktivkohle, $80 \mathrm{~W}$; 4: Quecksilbermanometer (300 Torr) ; 5: $\gamma$ Zählrohr; 6: Glas-Meßstutzen für das Zählrohr; 7: Durchführung für Thermoelement; 8: Kittstelle zwischen Glasapparatur und Pythagoras-Rohr 9: 10: Rohrofen für Temperaturen bis $1800{ }^{\circ} \mathrm{C}$; 11 : Platinfahne an der Lötstelle des Thermoelements; 12: Platinbehälter für die Quarzproben; 13: Thermoelement für die Temperaturregelung des Rohrofens.
\end{abstract}

ras-Masse ${ }^{2}$. Für die Kittstellen (7) und (8) wurde bei etwa $70^{\circ} \mathrm{C}$ erweichender weißer Siegellack verwendet. Das Thermoelement ( $\mathrm{Pt}-\mathrm{PtRh}$-Thermoelement, 0,35 $\mathrm{mm} \phi)$ trägt an der Lötstelle eine Platinfahne (11). Auf diese Fahne wird die in einem Platinbehälter befindliche Quarzprobe (12) gelegt. Der Probenbehälter verhindert, daß bei höheren Temperaturen das Quarzpulver an der Innenwand des Pythagoras-Rohres festsintert.

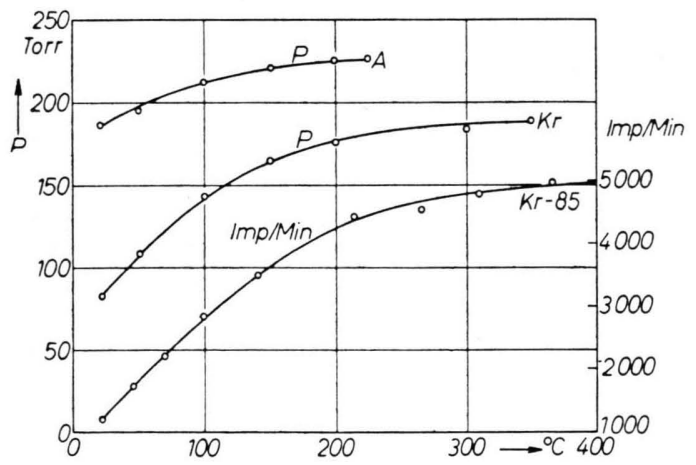

Abb. 2. Adsorptionskurven von Argon und Krypton an Aktivkohle. Eingefüllte Argonmenge 48,9 $\mathrm{cm}^{3}$ (NTP). Druck bei $18{ }^{\circ} \mathrm{C}$ in der $165 \mathrm{~cm}^{3}$ fassenden Anlage: 185 Torr. - Krypton-85 wurde am $\gamma$-Zählrohr 5 , Abb. 1 , gemessen. Die Aktivkohlemenge war in allen Fällen $10 \mathrm{~g}$. - Bei $-183^{\circ} \mathrm{C}$ ist die Adsorption vollständig.

Durch einen Rohrofen (10) ${ }^{3}$ läßt sich das Pythagoras-Rohr auf eine vorbestimmte Temperatur (zwischen 25 und $1600{ }^{\circ} \mathrm{C}$ ) heizen. Die maximale Aufheizgeschwin-

2 Hersteller: Fa. H a l d e n w a n g e r, Berlin. Zusammensetzung: $\mathrm{SiO}_{2}, \mathrm{Al}_{2} \mathrm{O}_{3}, \mathrm{KNaO}$; Schmelzpunkt: $1820^{\circ} \mathrm{C}$; Ausdehnungskoeffizient: $5,7 \cdot 10^{-6}$ (von $20^{\circ}-1000^{\circ} \mathrm{C}$ ); ziemlich gute Temperaturwechselbeständigkeit.

3 Rohrofen der Fa. H e ra e u s-Hanau mit Pt-Ir-Widerstandsheizung für max. $1800{ }^{\circ} \mathrm{C}$; geregelt wird mit einem digkeit von Zimmertemperatur bis $1000{ }^{\circ} \mathrm{C}$ beträgt etwa 40 min.

Das Volumen der Apparatur mit geschlossenen SтоскVentilen ist rd. $145 \mathrm{~cm}^{3}$.

\section{Eigenschaften und Behandlungsweise des Kryptons-85}

Das verwendete Krypton besteht zu $3 \%$ aus ${ }^{85} \mathrm{Kr}$ (Halbwertszeit 10,3 Jahre). Es ist in Glasampullen mit rd. $0,43 \mathrm{~cm}^{3}$ Gas (NTP) von einer Gesamtaktivität von $\mathrm{rd} .20 \mathrm{mC}$ erhältlich ${ }^{4}$.

Zur Füllung der Anlage mit dem Krypton wird die Ampulle statt des Pythagoras-Rohres in die Kittstelle (8) eingesetzt und die Apparatur durch das Sтоcк-Ventil (1 A) evakuiert. Dann schließt man (1 A) und zertrümmert die im Innern der Ampulle befindliche Glaskapillare mit einem magnetisch bewegten Stück Eisen. Nun öffnet man das Sтоск-Ventil (1 B) und adsorbiert das Krypton an der gekühlten Aktivkohle. Diese Adsorption verläuft sehr rasch, wie sich mit dem Zählrohr (5) feststellen läßt. Man schließt (1 B), wonach die Kühlung des Kohlebehälters nicht mehr erforderlich ist.

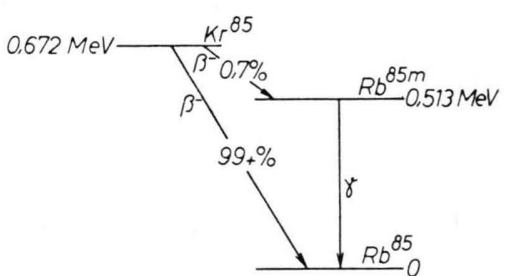

Abb. 3. Termschema des Krypton-85-Zerfalls ${ }^{5}$.

Ir-IrRh-Thermoelement (13) und Fallbügelinstrument. Die Regelschwankungen (Stark - Schwach-Regelung) betragen bei $1000^{\circ} \mathrm{C}$ höchstens $5^{\circ} \mathrm{C}$.

${ }^{4}$ Isotope-Division, Amersham-England.

5 D. Strominger, J. M. Hollander u. G.T. Seaborg, Rev. Mod. Phys. 30, 653 [1958]. 
Das Zerfallsschema von $\mathrm{Kr}-85$ ist in Abb. 3 dargestellt.

Krypton gefriert bei $-157^{\circ} \mathrm{C}$ und siedet bei $-152,9^{\circ} \mathrm{C}$. Die Gasdichte ist 3,708g/l (NTP).

\section{Versuchsführung}

Die Probe wird in den Platinzylinder (12) eingebracht. Während des Aufheizens evakuiert man über Ventil (1 A), um freiwerdende Gasreste abzusaugen. Erst nach Erreichen einer vorbestimmten Temperatur wird (1 A) geschlossen und das Ventil (1 B) zum Kryptonbehälter geöffnet. Schon vorher war die Aktivkohle in diesem Behälter auf $300{ }^{\circ} \mathrm{C}$ geheizt worden, so daß beim Öffnen des Ventils sofort die ganze Kryptonmenge zur Probe gelangen kann. Die eingelassene Gaskonzentration wird durch Impulsmessung am $\gamma$-Zählrohr (5) bestimmt.

Bei den Krypton-Einbauversuchen in Quarz wurde nach folgendem Programm verfahren: Die Quarzkristalle werden im Vakuum und von der Einlaßtemperatur (in den meisten Versuchen $700{ }^{\circ} \mathrm{C}$ ) an in Krypton aufgeheizt. Nach Erreichen der Versuchstemperatur $T_{x}$ wird die Temperatur für eine Zeit $t_{x}$ konstant gehalten, anschließend das Krypton wieder in die Aktivkohle adsorbiert und das Ventil (1 B) geschlossen. Jetzt hält man die Probe noch eine Stunde lang auf der gleichen Temperatur, um lose adsorbiertes Krypton z. B. aus Kristallritzen zu entfernen. In Abb. 4 ist zur Veranschaulichung das Temperatur - Zeit-Diagramm dieses Versuchsablaufs dargestellt.

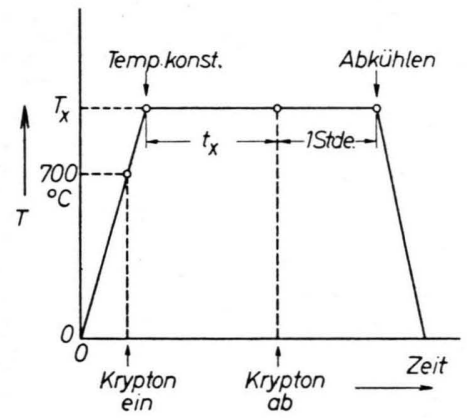

Abb. 4. Temperatur-Zeitdiagramm des Versuchsablaufs. $T_{x}$ Versuchstemperatur, $t_{x}$ Versuchszeit in der Kryptonatmosphäre. Aufheiz- und Abkühlungsgeschwindigkeit rd. $25^{\circ}$ pro min. Die Einlaßtemperatur für das Krypton war in den meisten Versuchen $700^{\circ} \mathrm{C}$.

Úber $1000{ }^{\circ} \mathrm{C}$ kommt es vor, daß der Druck in der Anlage etwas ansteigt. Das rührt entweder von Luft her, die durch das heiße Pythagoras-Rohr eindringen kann oder vom Gasen der Quarzprobe selbst. Der Anstieg beträgt bei 4 Stdn. Heizdauer rd. 3-4 Torr. Diese Fremdgasbeimengung stört erfahrungsgemäß den Einbau des Kryptons in den Quarzkristallen nicht.

\section{Aktivitätsmessung der Proben}

Nach dem Abkühlen wird die Probe entnommen und auf ihre Radioaktivität untersucht. Dies geschieht unter einem mit Blei abgeschirmten $\beta$-Endfensterzählrohr. Das aktive Quarzpulver ist in einem flachen Teller von rd. $25 \mathrm{~mm} \phi$ eben ausgebreitet. Der Abstand vom Zählrohrfenster betrug $18 \mathrm{~mm}$.

Bei der Versuchsführung waren vor allem bei feinkörnigem Quarz geringe Probenverluste unvermeidlich (höchstens $5 \%$ bei $1 \mathrm{~g}$ Substanz). Die Abhängigkeit der Zählrate einer Probe von ihrer Menge ist in Abb. 5 wiedergegeben. Aus ihr geht hervor, daß bereits ab $0,7 \mathrm{~g}$ (Korngröße 0,3 mm) geringe Unterschiede in der Substanzmenge für die Aktivitätsmessung unerheblich sind.

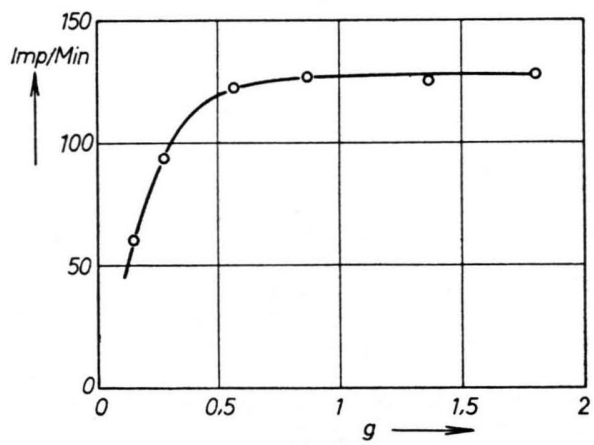

Abb. 5. Abhängigkeit der Zählrate (Imp./min) einer radioaktiven Quarzprobe von der Probenmenge unter dem $\beta$-Zählrohr. Korngröße $0,3 \mathrm{~mm}$.

Mit Hilfe eines radioaktiven Standards können geringe Schwankungen in der Zählrohrempfindlichkeit in den einzelnen Meßergebnissen korrigiert werden.

Die nachzuweisenden Impulsraten lagen in einem Bereich von 40 bis 1000 Impulsen/min über dem Nulleffekt, der bei der verwendeten Meßanordnung im Durchschnitt 17 Impulse/min betrug. Alle Messungen ließen sich durch eine einfache Umrechnung auf gleiche Gaskonzentration von radioaktivem Krypton [gemessen am $\gamma$-Zählrohr (6)] beziehen, da in einer Versuchsreihe gezeigt werden konnte, daß bei konstanter Versuchstemperatur Proportionalität zwischen der Gaskonzentration und der im Quarz eingebauten Kryptonmenge besteht. 


\section{Ergebnisse}

Als Ausgangsmaterial für die Untersuchung der Quarz - Cristobalit-Umwandlung wurde verwendet: A) Gekörnter Quarz (eisenfrei, für Analyse, Fa. R i e d e l-d e H a e n, Art. Nr. 31 622, Kristallquarz skandinavischen Ursprungs). Dieses Produkt (Korngröße $1-3 \mathrm{~mm}$ ) bestand zum überwiegenden Teil aus trüben Körnern, zum geringeren aus völlig klaren Kriställchen. Durch Aussuchen wurden die beiden Quarzsorten getrennt und jede für sich untersucht; B) Quarzeinkristall $6 \mathrm{~mm}$, Herkunft Brasilien; C) Quarzglaspulver.

Tab. 1 gibt die Bezeichnung mit Korngröße und Dichte der verwendeten Proben wieder.

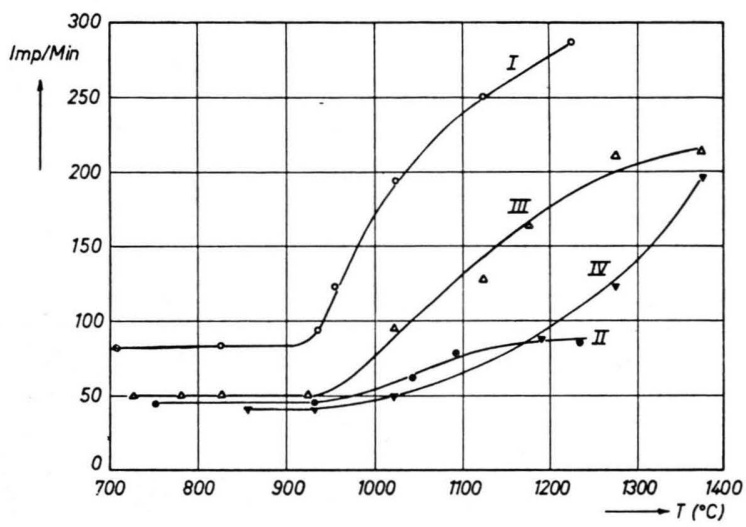

Abb. 6. Kryptoneinbau in kristallinem Quarz. I: trübe Quarzkörner (1-3 mm) ; II: klare Quarzkörner (1-3 mm); III: Quarzpulver $(0,30-0,58 \mathrm{~mm})$; IV: Quarzpulver $(0,065$ bis $0,21 \mathrm{~mm})$. Für jeden Meßpunkt wurde neues Ausgangsmaterial verwendet. 1 Std. Erhitzungsdauer in Kryptonatmosphäre.

\begin{tabular}{|c|l|c|c|}
\hline $\begin{array}{c}\text { Proben- } \\
\text { Nr. }\end{array}$ & Bezeichnung & $\begin{array}{c}\text { Durchmesser } \\
\mathrm{mm}\end{array}$ & $\begin{array}{c}\text { Dichte } \\
\mathrm{g} / \mathrm{cm}^{3}\end{array}$ \\
\hline I & trübe Quarzkörner & $1-3$ & 2,640 \\
II & klare Quarzkörner & $1-3$ & 2,649 \\
III & Quarzpulver* & $0,30-0,58$ & 2,640 \\
IV & Quarzpulver* & $0,065-0,21$ & 2,640 \\
V & Quarzglaspulver & $0,30-0,58$ & 2,203 \\
VI & Quarzeinkristalle & 6 & 2,649 \\
\hline
\end{tabular}

* Hergestellt aus unausgesuchtem Ausgangsprodukt A durch Zerkleinern in einem Achatmörser.

Tab. 1.

Bei allen Umwandlungsexperimenten im Temperaturbereich $700-1500^{\circ} \mathrm{C}$ war die Einlaßtemperatur des radioaktiven Kryptons $700{ }^{\circ} \mathrm{C}$. Die Versuchsdauer $t_{x}$ bei der jeweiligen Temperatur $T_{x}$ war 1 Stunde, die Ausheizdauer nach Entfernung des Kryptons ebenfalls 1 Stunde (s. Abb. 4). Die Aufheizgeschwindigkeit betrug rd. $25^{\circ} / \mathrm{min}$. Eingesetzt wurde für jeden Versuch genau $1 \mathrm{~g}$ Substanz.

In Abb. 6 sind die Ergebnisse an kristallinem Quarz dargestellt. Von Quarzglaspulver ist eine Versuchsreihe in Abb. 7 aufgetragen. Die Kryptonaktivität der erhitzten Proben ist in diesem Falle in einigen Bereichen der Kurve um mehr als 6-mal größer als bei den entsprechenden Versuchen mit kristallinem Quarzpulver. In Abb. 8 ist schließlich ein Vergleich zwischen Kryptonaufnahme und Dichteabnahme des eingesetzten Materials (Quarzpulver, $0,065-0,21 \mathrm{~mm})$ wiedergegeben. In dieser Versuchsreihe war die Einlaßtemperatur für Krypton wiederum $700{ }^{\circ} \mathrm{C}$, jedoch die Versuchsdauer $t_{x}$ in

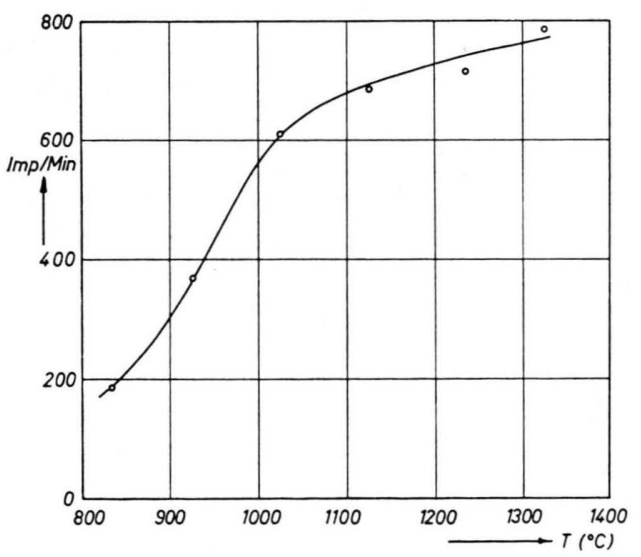

Abb. 7. Kryptoneinbau in pulverisiertem Quarzglas (0,30 bis $0,58 \mathrm{~mm})$. 1 Stde. Erhitzungsdauer in Kryptonatmosphäre.

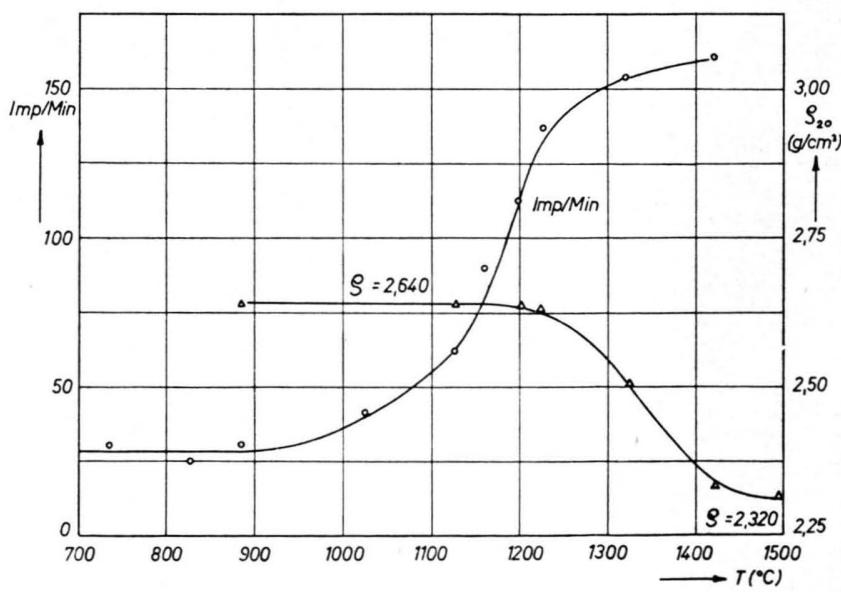

Abb. 8. Kryptoneinbau in kristallinem Quarzpulver $(0,065$ bis $0,21 \mathrm{~mm})$. $3 \mathrm{Stdn}$. Erhitzungsdauer in Kryptonatmosphäre. Nach Bestimmung der Zählrate wurden die gleichen Proben für Dichtemessungen $\left(\varrho_{20}\right)$ verwendet (rechte Ordinate). 
der Gasatmosphäre dreimal länger (3 Stdn.) als in den vorher aufgeführten Experimenten. Die Dichte der Proben wurde im Pyknometer mit Xylol als Flüssigkeitsfüllung bestimmt.

\section{Diskussion}

Vergleicht man die Radien von $\mathrm{Si}^{+4}, \mathrm{O}^{-2}$ und $\mathrm{Kr}$ (Tab. 2), so erkennt man, daß Diffusion des großen Kryptonatoms in einem $\mathrm{SiO}_{2}$-Kristall wenig wahrscheinlich ist.

\begin{tabular}{l|ll|}
\cline { 2 - 3 } Tab. 2. & $\mathrm{Kr}$ & $1,89 \AA$ (s. Anm. $\left.{ }^{6}\right)$ \\
Atom- bzw. & $\mathrm{Si}^{+4}$ & $0,38 \AA$ \\
Ionenradien. & $\mathrm{O}^{-2}$ & $1,45 \AA$ \\
\hline
\end{tabular}

Trotzdem findet man beim Erhitzen von Quarzpulver, daß bereits um $200{ }^{\circ} \mathrm{C}$ geringe Mengen von Krypton aus der Gasatmosphäre aufgenommen werden. Dieser Einbau ist jedoch wenig temperaturabhängig und erhöht sich auch kaum mit zunehmender Erhitzungszeit. Es kann sich somit nicht um eine echte Diffusion handeln. Vielmehr muß man annehmen, daß durch die Erwärmung im Quarzpulver vorhandene, stark gestörte Kristallebenen oder mikroskopische Risse rekristallisieren und das Krypton mechanisch einschließen ${ }^{7}$. Dafür spricht auch, daß dieses Krypton durch mehrstündiges Erhitzen im Vakuum bis zu $900{ }^{\circ} \mathrm{C}$ nicht mehr auszutreiben ist. In einem größeren Quarzeinkristall mit völlig intakten Wachstumsflächen konnte übrigens bis $900{ }^{\circ} \mathrm{C}$ keinerlei Krypton eingebaut werden.

Verfolgt man nach der beschriebenen Versuchsführung die Kryptonaktivität von Quarzkristallen bei steigender Versuchstemperatur, so findet man ab rd. $900{ }^{\circ} \mathrm{C}$ den in Abb. 6 dargestellten, sprunghaften Anstieg. Da die Kristalle ab $1200{ }^{\circ} \mathrm{C}$ deutlich trübe erscheinen, lag die Vermutung nahe, daß der Einbaueffekt ursächlich mit einer $\mathrm{SiO}_{2}$-Modifikationsänderung verbunden ist. In Betracht kommen in diesem Temperaturgebiet nur die Umwandlungen $\beta$-Quarz $\rightarrow \gamma$-Tridymit oder $\beta$-Quarz $\rightarrow \beta$-Cristobalit (Abb. 9). Die erstere ist jedoch noch nie ohne Fluß-

${ }^{6}$ R. T. SAnderson, J. Inorg. Nucl. Chem. 7, 288 [1958].

7 Ähnliche Untersuchungen über die Eindiffusion von radioaktivem Krypton in Anthraceneinkristalle bei $100^{\circ} \mathrm{C}$ ergeben eine ausgeprägte, autoradiographisch schön feststellbare Markierung von feinsten Haarrissen in den Kristallflächen. Das so eingebaute Krypton ließ sich ebenfalls erst bei höherer Temperatur wieder ausheizen. (Dipl.-Arbeit G. Lück, Techn. Hochschule, München 1960.) mittel beobachtet worden ${ }^{8-10}$. Es ist sogar zweifelhaft, daß Tridymit eine unter normalem Druck stabile Kristallphase des reinen $\mathrm{SiO}_{2}$ ist ${ }^{11}$. Es entsteht vielmehr zuerst immer $\beta$-Cristobalit ${ }^{12}$. Mit DebyeScherrer-Aufnahmen eines längere Zeit auf $1200{ }^{\circ} \mathrm{C}$ erhitzten Quarzpulvers konnte diese Cristobalitbildung eindeutig nachgewiesen werden. Auch die beobachtete Dichteänderung des Kristallpulvers von $\varrho_{20}=2,640$ ( $\alpha$-Quarz) auf $\varrho_{20}=2,33$ entsprach dieser Umwandlung $\left(\varrho_{20}\right.$ für Cristobalit $\left.=2,320\right)$.

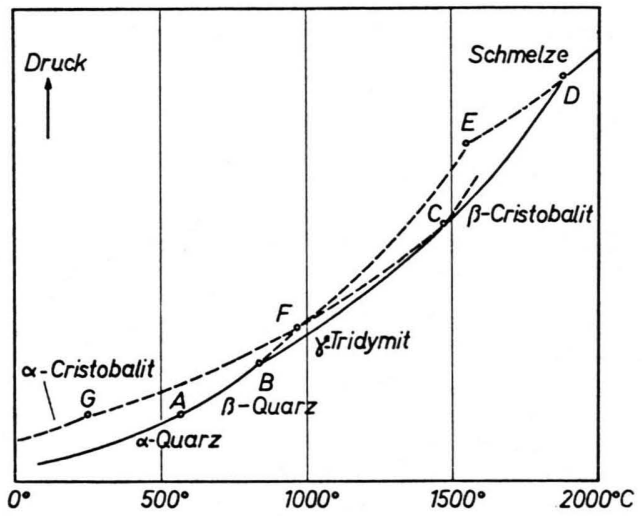

Abb. 9. Phasendiagramm der $\mathrm{SiO}_{2}$-Modifikationen (schematisch) ${ }^{24}$. Die angegebenen Umwandlungspunkte sind: A. $\alpha$ Quarz- $\beta$-Quarz; B. $\beta$-Quarz- $-\gamma$-Tridymit; C. $\gamma$-Tridymit $\beta$-Cristobalit; D. $\beta$-Cristobalit-Schmelze; E. $\beta$-QuarzSchmelze; F. $\beta$-Quarz $-\beta$-Cristobalit; G. $\alpha$-Cristobalit $-\beta$ Cristobalit. Nach $\mathrm{F}_{\text {LöRKE }}{ }^{11}$ ist in reinem $\mathrm{SiO}_{2} \beta$-Cristobalit die einzig stabile Phase im Temperaturbereich $\mathrm{F}-\mathrm{D}$, nicht $\gamma$-Tridymit.

Zweierlei Ursachen können für den Kryptoneinbau während der Phasentransformation verantwortlich gemacht werden:

1. Die Umwandlung Quarz - Cristobalit ist mit einer Volumenvermehrung um $\mathrm{rd} .15,5 \%$ verbunden. Hierdurch wird das ursprüngliche Gitter gesprengt; es entstehen zahlreiche Kriställchen, die durch Sinterung oder Rekristallisation Krypton einschließen können.

2. Während der Umwandlung Quarz - Cristobalit müssen Bindungen zwischen den einzelnen $\mathrm{SiO}_{4}$ Tetraedern gelöst werden, damit sich der Übergang von hexagonaler (Quarz) zu kubischer Kristall-

8 O. W. Flörke, Ber. dtsch. keram. Ges. 32, 369 [1955].

9 F. C. KRACEK, Phase Transformations in Solids, John Wiley, New York 1951, S. 269.

10 H. E. Schwiete u. H. Stollenwerk, Arch. Eisenhüttenw. 28, 17 [1957].

11 O. W. FLöRKE, Naturwiss. 18, 419 [1956].

12 N. N.Sinel'Nikov, C. R. Acad. Sci., USSR 102, 555 [1955]; Trans. Brit. Ceram. Soc. Abstr. 55, 986 [1956]. 
symmetrie ( $\beta$-Cristobalit) vollziehen kann (Phasenumwandlung mit Gitterneubau). In der Abb. $10 \mathrm{a}, \mathrm{b}$ sind schematisch diese beiden Strukturen dargestellt. Es bildet sich eine aufgelockerte Übergangsphase, die, wie neuerdings röntgenographische Untersuchungen zeigten ${ }^{13-15}$, bei der Quarz - Cristobalit-Umwandlung besonders stark ausgeprägt ist. Über $40 \%$ des ursprünglichen Quarzes können während der Transformation neben bereits entstandenem kristallinem Cristobalit als „röntgenamorphe“ Phase auftreten. Es ist nun anzunehmen, daß die Kryptonatome in dieses lockere Gefüge eindiffundieren können und während der weiteren Umwandlung und Rekristallisation fest eingebaut werden.
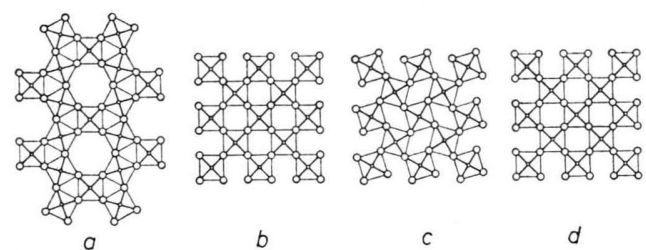

Abb. 10. Schematische Darstellung von Phasenumwandlungen mit Gitterneubau (a,b) und Gitterumbau (c, d) ${ }^{23}$. a zeigt eine hexagonale Grundform, die nur durch Bindungslösung in die kubische Anordnung b übergeführt werden kann; c ist die sog. $\alpha$ - oder Tiefform der Anordnung, die bei einer bestimmten Transformationstemperatur in die $\beta$ oder Hochform umklappt.

Verfolgt man die Phasenumwandlung von $\beta$ Quarz in $\beta$-Cristobalit einmal durch Einbau von Krypton, zum anderen durch Messung der Dichteabnahme, dann erhält man das bemerkenswerte Ergebnis, daß das Maximum der Änderungsgeschwindigkeit für beide Untersuchungsmethoden bei verschiedenen Temperaturen liegt (Abb. 8). Die Kryptonaufnahme eilt der Dichteänderung voraus.

Die Begründung hierfür ist folgende: Die Umwandlung beginnt nach Überschreiten der Umwandlungstemperatur nicht an beliebigen Stellen des Quarzgitters, sondern vorzugsweise an gestörten Kristallteilen, wie Korngrenzen, Versetzungen und vor allem Kristalloberflächen ${ }^{16,17}$. Krypton kann nur aufgenommen werden, während die Umwandlung an der Kristalloberfläche abläuft; tiefere Schichten sind für die großen Edelgasatome nicht erreichbar. Somit spricht die Kryptonaufnahme spezifisch

13 H. Müller-Hesse, H. E. Schwiete u. H. Stollenwerk, Naturwiss. 42, 437 [1955].

14 A. C. D. Chaklader u. A. L. Roberts, Trans. Brit. Ceram. Soc. 57, 115 [1958].

15 A. L. Roberts, Ber. dtsch. keram. Ges. 37, 397 [1960]. auf das Anfangsstadium der Umwandlung an und wird daher viel eher meßbar als die Änderung einer Volumeneigenschaft, wie es die summarische Dichte des Kristallgefüges ist.

Der Einbau an der Oberfläche ist im wesentlichen früher abgeschlossen als die Umwandlung des ganzen Kristallvolumens. Daher wird in Abb. 8 bei steigender Versuchstemperatur die Zunahme des Kryptoneinbaus schließlich immer geringer, während die Dichte sich noch erheblich ändert.

Aus Abb. 6 geht die Korngrößenabhängigkeit der Umwandlungsgeschwindigkeit hervor. Auffallend ist die wesentlich raschere Umwandlung der trüben Quarzkörner (I), im Vergleich zu den klaren (II). Für diese letzteren muß vermerkt werden, daß die Umwandlung in den einzelnen Körnern verschieden stark fortschritt und es bei $1200{ }^{\circ} \mathrm{C}$ in der Probe noch immer einen beträchtlichen Bruchteil völlig klarer, also nicht merklich in Cristobalit verwandelter Körner gab. Erst bei noch höheren Temperaturen waren praktisch alle Körner von der Reaktion betroffen. Die trüben Körner bestehen aus milchigtrübem Quarz, und ihre Umwandlung setzt rasch ab $900{ }^{\circ} \mathrm{C}$ in jedem Korn ein. Der Unterschied in der Umwandlungsgeschwindigkeit von reinem Bergkristall und Quarzit ist schon länger bekannt ${ }^{10,13}$. Trüber Quarz ist besonders reaktionsfähig und verhält sich mehr wie Quarzit.

$\mathrm{Zu}$ erwarten war nun, daß feinere Quarzproben wegen der größeren spezifischen Oberfläche sich schneller umwandeln und mehr Krypton aufnehmen als grobe Proben. Das ist um $900{ }^{\circ} \mathrm{C}$ jedoch nicht der Fall, weil die Umwandlung vorzugsweise von besonders aktiven Zentren ausgeht, die nicht in allen Körnern enthalten sind.

Nimmt man als grobe Näherung an, daß beim Zerkleinern eines Quarzkristalls die Zahl dieser besonders reaktionsfähigen Zentren nicht zunimmt, so erscheint das aktive Zentrum eines großen Kristalls nach dem Zerkleinern nur bei einem oder einigen wenigen der Bruchstücke. Nur diese Kriställchen können sich oberhalb $900^{\circ}$ rasch in Cristobalit umwandeln. Damit ist die Umwandlungsgeschwindigkeit einer solchen feinkörnigen Quarzprobe geringer als die einer grobkörnigen. - Bei höheren Temperaturen (über $1200{ }^{\circ} \mathrm{C}$ ) gibt es spontane Keimbildung; alle Kriställchen wandeln sich um und die Kryptonaufnahme steigt damit rasch an.

Zur weiteren Klärung des Umwandlungsverlaufs wurde an einem Einkristall mit natürlichen Wachstums-

16 N. N. Sinel'Nikov, Zapiski Vsesoyuz. Mineral. Obshchestva 87, 657 [1958]; Chem. Abstr. 53, 6920 [1959].

17 R. W. Grimshaw, J. Hargreaves u. A. L. Roberts, Trans. Brit. Ceram. Soc. 55, 36 [1956]. 
ebenen eine Fläche angeschliffen. Bis $900{ }^{\circ} \mathrm{C}$ war in diesem Kristall kein Krypton feststellbar. Nach vierstündigem Erhitzen bei $1250{ }^{\circ} \mathrm{C}$ ließ sich jedoch besonders in dieser gestörten Oberfläche eingelagertes Krypton nachweisen. An Autoradiographien konnte gezeigt werden, daß die Umwandlung von einigen wenigen Stellen der angeschliffenen Ebene ausging und sich von dort über die Kristalloberfläche und ins Innere ausbreitete.

Zur Sicherstellung, daß die Einsatztemperatur der Kryptonaufnahme bei $900{ }^{\circ} \mathrm{C}$ wirklich dem Transformationspunkt der Quarz-Cristobalit-Umwandlung entspricht, wurden drei Kriterien geprüft: a) Unabhängigkeit der Einsatztemperatur von der Erhitzungsdauer und b) von der Korngröße des Materials; c) Übereinstimmung mit der Einsatztemperatur der Rückumwandlung von Cristobalit in Quarz.

Die Lage der Einsatztemperatur des Kryptoneinbaus erwies sich tatsächlich als unabhängig von der Erhitzungsdauer. Knapp oberhalb $900{ }^{\circ} \mathrm{C}$ wurde auch bei mehrstündigem Erhitzen niemals ein Ansteigen der Radioaktivität des Kristallpulvers beobachtet. - Die Unabhängigkeit der Einsatztemperatur von der Korngröße geht aus Abb. 6 hervor. Zur Untersuchung der Rückumwandlung von Cristobalit in Quarz wurde feinstkörniges Cristobalit (hergestellt aus Quarzpulver durch 10-stündiges Erhitzen bei $1325^{\circ} \mathrm{C}$ ) in die Kryptonatmosphäre gebracht. Wie aus Tab. 3 ersichtlich ist, ergab sich wirklich ein Ansteigen des Einbaus zwischen 825 und $885^{\circ} \mathrm{C}$ (Rückumwandlung Quarz in Cristobalit). Unterhalb $825{ }^{\circ} \mathrm{C}$ blieb die Kryptonmenge konstant (die tiefe Temperatur gibt eine zu geringe Umwandlungsgeschwindigkeit). Über $1000{ }^{\circ} \mathrm{C}$ steigt der Kryptoneinbau infolge Sinterung und Rekristallisation des äußerst feinkörnigen Pulvers wieder an.

\begin{tabular}{|l|cccccc|}
\hline Temperatur & 825 & 885 & 925 & 985 & 1125 & ${ }^{\circ} \mathrm{C}$ \\
\hline Aktivität & 110 & 179 & 177 & 178 & 290 & Imp./min \\
\hline
\end{tabular}

Tab. 3. Temperaturabhängigkeit des Kryptoneinbaus in Cristobalit bei einstündiger Erhitzungsdauer.

Um $930{ }^{\circ} \mathrm{C}$ findet dagegen kein Fortschreiten des Einbaus statt. Das deutet darauf hin, daß bei dieser Temperatur die Umwandlungstendenz tatsächlich gering ist, wie man es in der Nähe eines (trägen) Umwandlungspunktes zu erwarten hat.

\footnotetext{
$18 \mathrm{Na}_{2} \mathrm{WO}_{4}$ als Flußmittel u. $68 \mathrm{Atm}$.; allmählicher Übergang des Quarzes zu fehlgeordnetem Cristobalit.

10 V.G. Hrll u. R. Roy, Trans. Brit. Ceram. Soc. 57, 496[1958].
}

Bisherige Bestimmungen des Umwandlungspunktes $\beta$-Quarz in $\beta$-Cristobalit sind in Tab. 4 zusammengefaßt.

\begin{tabular}{|c|c|c|c|}
\hline & $\begin{array}{l}\text { Trans- } \\
\text { formations- } \\
\text { temperatur } \\
{ }^{\circ} \mathrm{C}\end{array}$ & Methode & Autor \\
\hline 1. & $912^{18}$ & $\begin{array}{l}\text { RöNTGEN- } \\
\text { Analyse }\end{array}$ & $\begin{array}{l}\text { V. G. Hill }(1958)^{19} \\
\text { R. Roy }\end{array}$ \\
\hline 2. & ca. 1050 & $\begin{array}{l}\text { RöNTGEN- } \\
\text { Analyse }\end{array}$ & $\begin{array}{l}\text { O. W. FLÖRKE } \\
(1956)^{11}\end{array}$ \\
\hline 3. & $890-900^{20}$ & $\begin{array}{l}\text { RöNTGEN- } \\
\text { Analyse }\end{array}$ & S. S. CoLE $(1935)^{21}$ \\
\hline 4. & 950 & Dichte & $\begin{array}{l}\text { J. vaN NIEUWEN- } \\
\text { BURG }(1929)^{22}\end{array}$ \\
\hline 5. & $<1270$ & DTA & $\begin{array}{l}\text { R. W. GRIMSHAW } \\
(1956)^{17} \\
\text { J. HARGREAVES } \\
\text { A. L. ROBERTS }\end{array}$ \\
\hline 6. & $930 \pm 15$ & $\begin{array}{c}\text { Krypton- } \\
\text { einbau }\end{array}$ & diese Arbeit (1960) \\
\hline
\end{tabular}

Tab. 4. Transformationstemperatur der Quarz-CristobalitUmwandlung.

Es ist zu beachten, daß die früheren Bestimmungen ausschließlich mit volumenempfindlichen Methoden, wie Dichteänderung, Differentialthermoanalyse, Röntgen-Analyse, optischer Analyse ausgeführt worden sind. Das erklärt zum Teil die größere Schwankungsbreite und beim trägen Umwandlungsverlauf der Cristobalitbildung vor allem die Tendenz zu einer zu hohen Umwandlungstemperatur. Die Kryptonmethode erfaßt dagegen empfindlich den Beginn der Transformation, die daher auch in wesentlich kürzerer Versuchszeit nachweisbar wird.

In Verbindung mit diesen Untersuchungen an kristallinem Quarz sind entsprechende Versuche an Quarzglas von Interesse. Möglicherweise ist die "röntgenamorphe Zwischenphase" bei der Quarz Cristobalit-Umwandlung (s. o.) in ihren Eigenschaften vergleichbar mit der ebenfalls amorphen und offenen Beschaffenheit eines Quarzglases.

In Abb. 7 ist die Kryptonaufnahme von gepulvertem Quarzglas (Korngröße 0,30-0,58 mm) wiedergegeben. Die Radioaktivität ist erheblich höher als in kristallinem Quarzpulver von vergleichbarer Körnung (Abb. 6, Kurve III). Dieses Verhalten weist auf die überragende Bedeutung einer amorphen Struktur für die Kryptonaufnahmefähigkeit hin.

\footnotetext{
20 Mit Natriumsilikat als Flußmittel.

21 S. S. Cole, J. Amer. Ceram. Soc. 18, 149 [1935].

22 J.van Nieuwenburg, Rec.Trav.Chim.Pays-Bas 48,402 [1929].
} 


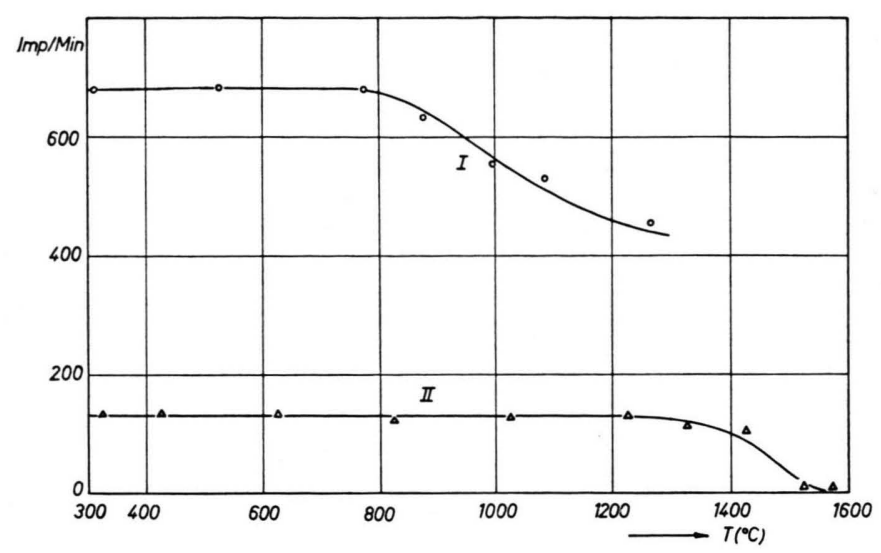

Abb. 11. Ausheizkurven. I: Quarzglas ( 0,30 bis $0,58 \mathrm{~mm}$ ). Als Ausgangsprobe wurde $1 \mathrm{~g}$ Quarzglaspulver verwendet mit 1 Stde. Erhitzungsdauer in Kryptonatmosphäre bei $1100^{\circ} \mathrm{C}$ und 1 Stde. Erhitzungsdauer im Vakuum. Diese Probe wurde jeweils 1 Stde. bei fortschreitend gesteigerten Temperaturen an der Luft ausgeheizt. - II: Quarzprobe $(1-3 \mathrm{~mm})$. Erhitzungsdauer in Kryptonatmosphäre $3 \mathrm{Stdn}$. bei $1200^{\circ} \mathrm{C}$ und 1 Stde. Erhitzungsdauer im Vakuum. Diese Probe wurde jeweils bei fortschreitend gesteigerter Temperatur an der Luft ausgeheizt.
Über $1200^{\circ} \mathrm{C}$ werden die Körner des Glaspulvers allmählich trübe. Die Dichte steigt von 2,203 des Ausgangsmaterials auf $2,235 \mathrm{~g} / \mathrm{cm}^{3}$; bei $1300{ }^{\circ} \mathrm{C}$ wird bereits $\varrho_{20}=2,320 \mathrm{~g} / \mathrm{cm}^{3}$ erreicht. Letzterer Wert entspricht der Dichte von Cristobalit. Es liegt also auch hier eine träge Phasenumwandlung zu Cristobalit vor, diesmal jedoch verbunden mit einer Volumenabnahme um 5,3\%.

Bemerkenswert ist ferner, da $\beta$ das aufgenommene Krypton nur ganz allmählich - und zwar erst bei sehr hoher Temperatur - wieder aus dem Pulver entweichen kann. Dieses Verhalten widerspricht einer echten Diffusion beim Einbau des Gases. In Abb. 11, Kurve I, ist die Ausheizkurve einer Quarzglasprobe dargestellt, die zuvor eine Stunde bei $1100{ }^{\circ} \mathrm{C}$ in der Kryptonatmosphäre erhitzt worden war. Die Meßpunkte in dieser Kurve sind Temperaturwerte, bei denen das Pulver anschließend eine Stunde lang an der Luft erhitzt wurde.

An einigen Quarz- bzw. Cristobalitproben wurden ebenfalls Ausheizuntersuchungen angestellt. Ein Beispiel ist in Abb. 11, Kurve II, wiedergegeben. Das Quarzpulver (Korngröße 1-3 mm, Menge $2 \mathrm{~g}$ ) war zuvor drei Stunden bei $1200{ }^{\circ} \mathrm{C}$ in der Kryptonatmosphäre erhitzt worden. Es hat den Anschein - das zeigen auch eine Reihe anderer Ausheizversuche - , daß das eingebaute Krypton hauptsächlich erst dann im Kristall beweglich wird und entweichen kann, wenn im betreffenden Gitter eine neue Phasenumwandlung (mit Gitterneubau) vor sich geht (z. B. Übergang zu einer Schmelze). Bei tiefer Tempera-

23 M. J. Buerger, Phase Transformations in Solids, John Wiley, New York 1951, S. 183.

24 C. N. FENNER, Amer. J. Sci. (4) 36, 331 [1913]; Z. anorg. Chem. 85, 133 [1914]. tur ist das Krypton so fest gebunden, daß sich das radioaktive Quarzpulver ohne weiteres als "festes“ Krypton-85-Präparat für Eich- und Meßzwecke verwenden läßt.

Mit derselben Versuchstechnik wurden schließlich auch die Umwandlungen $\alpha \gtrless \beta$-Quarz und $\alpha \rightleftarrows \beta$ Cristobalit untersucht. Diese Phasenumwandlungen haben in beiden Fällen keinerlei Einfluß auf die Kryptonaufnahme.

\begin{tabular}{|c|c|c|c|}
\hline $\begin{array}{c}\text { Um- } \\
\text { wandlung }\end{array}$ & $\begin{array}{c}\text { Trans- } \\
\text { formations- } \\
\text { punkt }{ }^{\circ} \mathrm{C}\end{array}$ & $\begin{array}{c}\text { Vol.- } \\
\text { Änderung }\end{array}$ & Kristallsystem \\
\hline $\begin{array}{l}\alpha \rightarrow \beta- \\
\text { Quarz }\end{array}$ & $\begin{array}{l}573^{23} \\
575^{24,25}\end{array}$ & $0,83 \%$ & $\begin{array}{l}\text { trigonal- } \\
\text { trapezoedrisch } \\
\rightarrow \text { hexagonal- } \\
\text { trapezoedrisch }\end{array}$ \\
\hline $\begin{array}{l}\alpha \rightarrow \beta- \\
\text { Cristoballit }\end{array}$ & $\begin{array}{l}260-270^{26} \\
235-268^{27}\end{array}$ & $2,8 \%$ & $\begin{array}{l}\text { tetragonal } \rightarrow \\
\text { kubisch }\end{array}$ \\
\hline
\end{tabular}

Tab. 5. $\alpha \gtrless \beta$-Umwandlungen von Quarz und Cristobalit.

In Tab. 5 sind einige charakteristische Daten zusammengestellt. Die schnellen $\alpha \rightleftarrows \beta$-Transformationen unterscheiden sich in ihrem Mechanismus grundsätzlich von der oben behandelten trägen Quarz Cristobalit-Umwandlung: es werden die Gitterbaueinheiten (hier die $\mathrm{SiO}_{4}$-Tetraeder) durch einen Umklappvorgang nur hinsichtlich der Valenzwinkel gegeneinander verdreht (Phasenumwandlung mit Gitterumbau), ohne daß Bindungen gelöst werden müssen. Schematisiert ist ein solcher Übergang in

25 J. van Nieuwenburg u. H. J. Zijlstra, Rec. Trav. Chim. Pays-Bas 47, 1 [1928].

26 O. W. FlöRKE, Ber. dtsch. keram. Ges. 34, 343 [1957].

27 O. Krisement u. G. Trömel, Z. Naturforschg. 14 a, 912 [1959]. 
Abb. $10 \mathrm{c}, \mathrm{d}$ dargestellt ${ }^{28}$. Es bildet sich während der Umlagerung keine aufgelockerte Übergangsphase wie bei Quarz $\rightarrow$ Cristobalit; die großen Kryptonatome können nicht in das Gitter eindringen.

${ }^{28}$ Für die $\alpha \rightleftarrows \beta$-Quarz-Transformation siehe auch die maßstabsgetreue Darstellung bei Anm. ${ }^{29}$.
Wir danken Herrn Prof. Dr. N. RieHL sehr für die Förderung dieser Arbeit. - Frl. R. Schumann und Herrn H. Bradatsch sind wir für die Ausführung zahlreicher Messungen zu Dank verpflichtet.

29 Landolt-Börnstein, Zahlenwerte u. Funktionen, Bd. 1, Teil 4 [1955], S. 35 .

\title{
Zur Thermolumineszenz von $\mathrm{MgF}_{2} / \mathrm{Mn}$
}

\author{
Von P. Bräunlich, W. Hanle und A. Scharmann \\ Aus dem Physikalischen Institut der Universität Gießen \\ (Z. Naturforschg. 16 a, 869—872 [1961] ; eingegangen am 30. Mai 1961) \\ Herrn Prof. Dr. N. Riehl zum 60. Geburtstag gewidmet
}

\begin{abstract}
Die Thermolumineszenz von $\mathrm{MgF}_{2} / \mathrm{Mn}$ nach Anregung mit Röntgen-Strahlung wird als Funktion des Aktivatorgehaltes untersucht und die Eignung zur Dosimetrie diskutiert.
\end{abstract}

Die Thermolumineszenz anorganischer Leuchtstoffe hat in letzter Zeit in der Dosimetrie eine gröBere Bedeutung gewonen. Wenn man Leuchtstoffe mit ionisierender Strahlung bestrahlt, können Elektronen in das Leitfähigkeitsband gehoben und dann von Haftstellen (traps) eingefangen werden. Führt man der Probe Energie zu, dann werden die Haftstellen entleert, und die freigemachten Elektronen können zur Lumineszenz Anlaß geben. Wird die Energie in thermischer Form zugeführt, spricht man von Thermolumineszenz. Der Intensitätsverlauf der Lumineszenz bei Aufheizen mit konstantem Temperaturgradienten heißt Glow-Kurve. Zur Dosimetrie können sowohl die Gesamtlumineszenzintensität (Lichtsumme) als auch die Höhe einzelner Peaks der Glow-Kurve herangezogen werden.

Bisher sind eine ganze Reihe von Versuchen gemacht worden, die Energiespeicherung in Leuchtstoffen zur Dosismessung auszunutzen. Dabei wurde nach einem Leuchtstoff gesucht, der folgenden Anforderungen genügt: hohe Nachweisempfindlichkeit

1 F. Daniels, C. A. Boyd u. D. F. SAunders, Science 117, 343 [1953].

2 W. Kossel u. U. Mayer, Naturwiss. 43, 4 [1956].

3 J. Ginther u. R. D. Kirk, J. Electrochem. Soc. 104, 365 [1957].

4 W. Hanle u. H. Peter, Ber. d. Oberhess. Ges. f. Natur- und Heilkunde 29, 105 [1958].

5 D. A. Patterson u. H. Friedman, J. Opt. Soc. Amer. 47, 1136 [1957].

6 H. Peter, Atomkernenergie 5, 453 [1960].

7 F. G. Houtermans, E. Jäger, M. Schön u. H. Stauffer, Ann. Phys., Lpz. 20, 283 [1957]. herab bis ins Milliröntgen-Gebiet, Speicherfähigkeit der Energie über einige Wochen ohne merkliches Ausbleichen, Dosis-proportionale Anzeige und sogenannte Wellenlängenunabhängigkeit. Wellenlängenunabhängigkeit bedeutet, daß sich der Leuchtstoff bezüglich seiner Absorption der RöNTGEN- und $\gamma$-Strahlung wie Luft verhält. Hierfür muß im Bereich der Photo- und Compton-Absorption die sogenannte effektive Ordnungszahl des Leuchtstoffes gleich der von Luft $\left(z_{\text {eff }}=7,7\right)$ sein.

Auf ihre Eignung als Dosimetersubstanz wurden bisher eine ganze Reihe von Leuchtstoffen untersucht ${ }^{1-10}$. Besonders gute Eigenschaften besitzt das $\mathrm{CaF}_{2} / \mathrm{Mn}^{8-10}$. Auch wird das CalciumfluoridThermolumineszenz-Dosimeter schon technisch hergestellt ${ }^{*}$. Allerdings beträgt seine effektive Ordnungszahl $z_{\text {eff }}=16,3$. Im folgenden sollen die Eigenschaften von $\mathrm{MgF}_{2} / \mathrm{Mn}$ untersucht werden, dessen effektive Ordnungszahl $\left(z_{\text {eff }}=10\right)$ besser mit der von Luft übereinstimmt.

8 M. ScнöN, Vorträge auf der Tagung der Schutzkommission der Deutschen Forschungsgemeinschaft in Karlsruhe am 29. - 30. 5. 1959 und dem Symposium über Dosimetrie der IAEA in Wien am 7.-11. 6. 1960.

9 J. H. Schulman, R. J. Ginther, R. D. Kirk u. S. Goulart, Nucleonics 18, 12 (März) [1960].

10 J. H. Schulman, F. H. Atrix, E. J. West u. R. J. Ginther. Rev. Sci. Instrum. 31, 1263 [1960].

* Manufacture Belge de Lampes et de Materiel Electronique, Brüssel. 